\section{Informations- und Aufklärungspflichten des Radiologen vor Durchführung einer einzelnen Röntgenaufnahme}

\section{Einleitung}

Die Frage, ob und in welchem Umfang Radiologen die Verpflichtung trifft, einen Patienten über Risiken einer einzelnen Röntgenaufnahme aufzuklären, war bereits in der Vergangenheit Gegenstand mehrerer Beiträge in der Rubrik „Radiologie und Recht“.

Mit dem Gesetz zur Neuordnung des Rechts zum Schutz vor der schädlichen Wirkung ionisierender Strahlung vom 27.06.2017², das am 31.12.2018 in Kraft getreten ist, wurde in Umsetzung der Richtlinie 2013/59/Euratom $^{3}$ nicht nur das Strahlenschutzgesetz (StrISchG) vollständig neu gefasst, sondern auch die Grundlage dafür geschaffen, die zuvor in der Röntgenverordnung (RöV) ${ }^{4}$ und der (alten) Strahlenschutzverordnung (StrlSchV5, im Folgenden: a. F.) enthaltenen Regelungen in der neuen Strahlenschutzverordnung $\left(\right.$ StrlSchV) ${ }^{6}$ zusammenzuführen. Eine Differenzierung nach der Quelle ionisierender Strahlung, ob natürlichen bzw. zivilisatorischen Ursprungs (vormals Anwendungsbereich der StrlSchV a. F.) oder Strahlung einer Röntgeneinrichtung oder eines Störstrahlers

1 Vgl. insbesondere Wigge/Loose, Ärztliche Aufklärungspflichten bei diagnostischen Röntgenuntersuchungen, 1. Teil, RöFo 2/2016, $218 \mathrm{ff}$. und 2. Teil, RöFo 3/2016, $312 \mathrm{ff}$.; Wigge/Kirsch, RöFo 9/2019, 873 ff.

2 BGBI. I, S. 1966

3 Richtlinie 2013/59/Euratom des Rates vom 5. Dezember 2013 zur Festlegung grundlegender Sicherheitsnormen für den Schutz vor den Gefahren einer Exposition gegenüber ionisierender Strahlung, ABI. L 13/1 vom 17.01.2014, S. 1.

$4 \quad$ Neugefasst durch Beschluss vom 30.04.2003 (BGBI. I, S. 604), zuletzt in der geänderten Fassung der Verordnung zur Änderung strahlenschutzrechtlicher Verordnungen vom 04.10.2011 (BGBI. I, S. 2000).

5 Verordnung für die Umsetzung der EURATOM-Richtlinien zum Strahlenschutz vom 20.07.2001 (BGBI. I, S. 1714), zuletzt geänderte Fassung vom 22.04.2002 (BGBI. I, S. 1459).

$6 \quad$ Verordnung zur weiteren Modernisierung des Strahlenschutzrechts vom 29.11.2018 (BGBI. I, S. 2034). (vormals Anwendungsbereich der RöV), erfolgt nicht mehr. Für Radiologen gelten heute einheitlich die Vorschriften des neuen StrISchG und der neuen StrISchV.

Im Hinblick auf den vorgenannten Regelungskomplex stellt sich die den Kern dieses Beitrags bildende und für die alltägliche radiologische Praxis höchst relevante Frage, ob mit der Änderung der Rechtslage eine weitergehende Verpflichtung des Arztes einhergeht, Patienten vor der Durchführung einer einzelnen Röntgenuntersuchung über Folgen und Risiken der ionisierenden Strahlung aufzuklären.

\section{Informationspflichten nach der (neuen) Strahlenschutz- verordnung}

Die Pflichten, die Radiologen im Zusammenhang mit der Durchführung einer Röntgenuntersuchung treffen, ergeben sich aus den $§ 119 \mathrm{ff}$. StrlSchV. Ein erster Anhaltspunkt hinsichtlich einer strahlenschutzrechtlichen Aufklärungspflicht des Radiologen gegenüber dem Patienten könnte sich aus $§ 124$ StrlSchV ergeben, dessen Absatz 1 eine grundsätzliche Pflicht zur Information des Patienten über Folgen und Risiken ionisierender Strahlung normiert, im Wortlaut (sämtliche Hervorhebungen im Dokument durch die Verfasser):

„Der Strahlenschutzverantwortliche hat dafür zu sorgen, dass eine Person, an der ionisierende Strahlung oder radioaktive Stoffe angewendet werden, vor der Anwendung über das Risiko der Strahlenanwendung informiert wird. "

Nach dem eindeutigen Wortlaut trifft den Strahlenschutzverantwortlichen eine Informationspflicht gegenüber dem Patienten. Es verbleiben jedoch Unklarheiten hinsichtlich der Umsetzung dieser Informationspflicht in der täglichen Praxis. Einerseits enthält §124 Abs. 1 StrlSchV keine Vorgaben bezüglich der ordnungsgemäßen Erfüllung der normierten Informationspflicht. Andererseits ergibt sich - jedenfalls

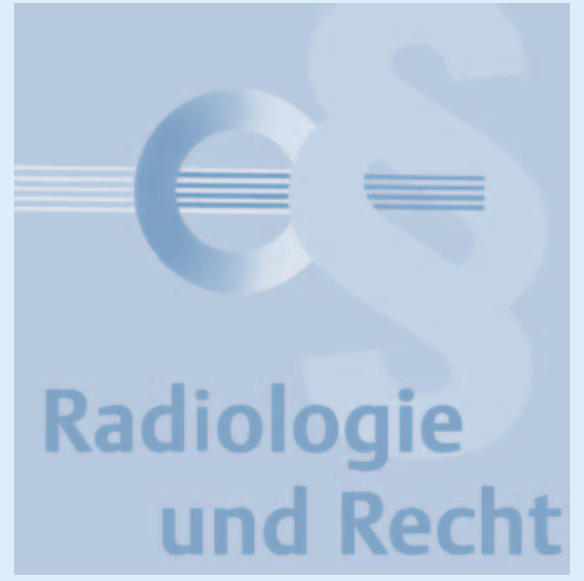

aus dem Wortlaut - nicht, über welche fachlichen Qualifikationen der Strahlenschutzverantwortliche verfügen muss und ob der Arzt selbst Adressat der Informationspflicht ist, oder ob er diese delegieren kann.

Um Sinn und Inhalt des §124 StrISchV näher zu ergründen ist zunächst die Begründung zur StrISchV heranzuziehen. Dort wird $\S 124$ unter anderem in folgender Weise kommentiert:

„Die nach Absatz 1 vorzunehmende Information zum Risiko der Strahlenanwendung erfordert keinen Mehraufwand, da bereits $\$ 630 c$ Absatz 2 Satz 1 des Bürgerlichen Gesetzbuchs - BGB den behandelnden Arzt zu Beginn der Behandlung zu einer ausführlichen und für den Patienten verständlichen Aufklärung verpflichtet. “7

Die Verordnungsbegründung verweist zwar ausdrücklich auf §630c Abs. 2 S. 1 BGB, der seinerseits Informationspflichten als Verpflichtungen des Behandlers im Rahmen des Behandlungsvertrages nach §630a BGB normiert. Andererseits greift sie jedoch den Begriff der „Aufklärung“ auf, der grundsätzlich mit den in $§ 630$ e Abs. 1 und 2 BGB festgeschriebenen zivilrechtlichen Verpflichtungen in Verbindung zu bringen ist. Damit bleibt auf den ersten Blick unklar, woran sich die strahlenschutzrechtliche Informationspflicht orientiert.

Zur Aufklärung der vorgenannten Fragestellung ist es hilfreich, zunächst die wesentlichen Verpflichtungen eines Radiologen, die

$7 \quad$ BR-Drs. 423/18, S. $317 \mathrm{f}$ 
im Zusammenhang mit der Durchführung einer Röntgenaufnahme nach der angesprochenen Neuordnung des Strahlenschutzrechts zu erfüllen sind, näher zu beleuchten. In einem weiteren Schritt werden die in der eben zitierten Begründung zur StrISchV aufgegriffenen zivilrechtlichen Vorschriften gesondert betrachtet.

\section{Wesentliche Pflichten im}

\section{Zusammenhang mit der} Durchführung einer Röntgenaufnahme nach der Neuordnung des Strahlenschutzrechts

\section{a. Stellung der rechtfertigenden Indikation durch den Arzt}

Der strahlenschutzrechtliche Rahmen der Anwendung ionisierender Strahlung am Menschen ergibt sich aus der zentralen Vorschrift des $\S 83$ StrlSchG. Darin findet sich auch das - bereits in der Vorgängervorschrift des §23 Abs. 1 S. 2 RöV enthaltene Prinzip der Einzelfallabwägung ${ }^{8}$, dem zufolge jeder Anwendung ionisierender Strahlung am Menschen ein individueller Abwägungsund Rechtfertigungsprozess des Arztes vorauszugehen hat. So heißt es wörtlich in $\S 83$ Abs. 3 S. 1 und 2 StrlSchG:

„Die Anwendung darf erst durchgeführt werden, nachdem ein Arzt oder Zahnarzt mit der erforderlichen Fachkunde im Strahlenschutz entschieden hat, dass und auf welche Weise die Anwendung durchzuführen ist (rechtfertigende Indikation). Die rechtfertigende Indikation erfordert bei Anwendungen im Rahmen einer medizinischen Exposition die Feststellung, dass der gesundheitliche Nutzen der einzelnen Anwendung gegenüber dem Strahlenrisiko überwiegt."

Die Maßstäbe, die der Arzt an die durchzuführende Abwägung anzulegen hat, werden in $\S 83$ Abs. 2 StrlSchG, der inhaltlich dem $\S 2$ a Abs. 2 RöV entspricht ${ }^{9}$, wie folgt näher beschrieben:

8 Vgl. auch BT-Drs. 18/11241, S. 334.

9 Vgl. auch a. a. O., S. 333.
„Die Anwendung muss einen hinreichenden Nutzen erbringen. Bei der Bewertung, ob die Anwendung einen hinreichenden Nutzen erbringt, ist ihr Gesamtpotential an diagnostischem oder therapeutischem Nutzen, einschließlich des unmittelbaren gesundheitlichen Nutzens für den Einzelnen und des Nutzens für die Gesellschaft, gegen die von der Exposition möglicherweise verursachte Schädigung des Einzelnen abzuwägen."

Diesbezüglich ist fraglich, inwieweit der Arzt zum Zwecke der Stellung der rechtfertigenden Indikation vor der Durchführung der Strahlenanwendung bzw. konkret der Röntgenaufnahme mit dem Patienten persönlich in Kontakt treten muss. Hierzu äußert sich §83 Abs. 3 S. 4 StrlSchG:

„Die rechtfertigende Indikation darf nur gestellt werden, wenn der Arzt, der die Indikation stellt, die Person, an der ionisierende Strahlung oder radioaktive Stoffe angewendet werden, vor Ort persönlich untersuchen kann, es sei denn, es liegt ein Fall der Teleradiologie nach $\S 14$ Absatz 2 vor."

Bereits der vorgenannte Wortlaut deutet an, dass der Arzt, der beabsichtigt, eine Röntgenaufnahme bei einem Patienten durchzuführen, grundsätzlich in der Lage sein muss, den Patienten vor der Durchführung zu untersuchen. Dass eine solche Untersuchung verpflichtend ist, ist der gesetzlichen Formulierung („untersuchen kann“) jedoch nicht zu entnehmen.

Diese Auslegung wird auch durch aktuelle und vorangegangene Verordnungs- bzw. Gesetzesbegründungen gestützt. Bereits zu der inhaltlich entsprechenden Vorgängervorschrift aus §23 Abs. 1 S. 5 RöV hieß es ausdrücklich:

„Absatz 1 stellt klar, dass der Arzt mit der erforderlichen Fachkunde im Strahlenschutz, der die rechtfertigende Indikation stellt, grundsätzlich die Möglichkeit haben muss, den Patienten unmittelbar zu untersuchen. Da eine persönliche Untersuchung durch den Arzt nach § 24 Abs. 1 Nr. 1 oder 2 vor Anfertigen einer Röntgenaufnahme in eindeutigen Fällen nicht immer erforderlich erscheint, wurde eine generelle Pflicht zur Untersuchung nicht festgelegt. Satz 5 verdeutlicht, dass im Unterschied zur Teleradiologie der Arzt mit der erforderlichen Fachkunde im
Strahlenschutz sich grundsätzlich in räumlicher Nähe zum Patienten aufhalten und damit jedenfalls die Möglichkeit gegeben sein muss, dass er sich persönlich mit dem Patienten befasst. "10

Dass diesbezüglich keine Änderung eingetreten ist, wird durch die Gesetzesbegründung zum neuen $\S 83$ Abs. 3 S. 4 StrISchG deutlich. Der Gesetzgeber hat in der Begründung nicht allein klargestellt, dass die Regelung inhaltlich der Vorschrift des $\$ 23$ Abs. 1 S. 5 RöV entspricht, sondern darüber hinausgehend die Ausgestaltung der Untersuchungspflicht des Arztes beschrieben und die für die Vorschrift maßgeblichen Motive erläutert:

„Das Stellen der rechtfertigenden Indikation setzt hinreichendes Wissen über den Gesundheitszustand der Person, an der ionisierende Strahlung oder radioaktive Stoffe angewendet werden, voraus. Dieses kann nicht in allen Fällen nur anhand von Unterlagen erfolgen, sondern kann auch eine persönliche Befragung und Untersuchung der Person erfordern. Hierzu ist die Anwesenheit des Arztes mit der erforderlichen Fachkunde im Strahlenschutz am Untersuchungs- oder Behandlungsort bei der Indikationsstellung zu fordern. Diese Anforderung ist eng auszulegen, damit er sich auch bei Untersuchungen mit zeitlichem Druck erforderlichenfalls mit dieser Person befassen und den Untersuchungsablauf für den Einzelfall festlegen kann. Allein bei Untersuchungen im Rahmen der Teleradiologie nach \& 14 Absatz 2 kann von diesem Grundsatz abgewichen werden."11

Damit steht im Ergebnis fest, dass der Arzt, der die rechtfertigende Indikation stellt, nicht grundsätzlich zu einer persönlichen Befragung und Untersuchung des Patienten vor Ort vor Durchführung einer ärztlichen Maßnahme, die mit der medizinischen Anwendung ionisierender Strahlen oder radioaktiver Stoffe verbunden ist, verpflichtet ist. Ergeben sich keine Anhaltspunkte dafür, dass die Maßnahme für den Patienten im individuellen Einzelfall mit einem besonderen Risiko verbunden ist, ist der Arzt nicht verpflichtet, eine persönliche Untersuchung durchzuführen.

\footnotetext{
10 BR-Drs. 230/02, S. 92.

11 BT-Drs. 18/11241, S. 334.
} 
Im Rahmen der zu treffenden Einzelfallabwägung und der anschließenden Durchführung der Maßnahme hat der Arzt zudem insbesondere das strahlenschutzrechtliche Gebot der Optimierung - auch als Vermeidungsoder Reduzierungsgebot bezeichnet - zu berücksichtigen, das bereits in $\S 25$ Abs. 2 S. 1 und 2 RöV niedergelegt war ${ }^{12}$ und auch nach der Neuordnung des Strahlenschutzrechts weiter zu beachten ist. Der neue $\S 83$ Abs. 5 StrlSchG sieht diesbezüglich vor:

„Die Exposition durch eine Untersuchung mit ionisierender Strahlung oder radioaktiven Stoffen ist so weit einzuschränken, wie dies mit den Erfordernissen der medizinischen Wissenschaft zu vereinbaren ist. Bei der Anwendung ionisierender Strahlung oder radioaktiver Stoffe zur Behandlung von Menschen ist die Dosis außerhalb des Zielvolumens so niedrig zu halten, wie dies unter Berücksichtigung des Behandlungsziels möglich ist."

\section{b. Weitere Pflichten des Radiologen im Kontext der Stellung der rechtfertigenden Indikation}

Eine nähere Konkretisierung der Anforderungen an die Vorgehensweise des Arztes im Rahmen der Stellung der rechtfertigenden Indikation erfolgt, wie bereits angemerkt, durch die $\S 119 \mathrm{ff}$. StrlSchV. Nach $\S 119$ Abs. 1 StrlSchV hat der Arzt zu prüfen, ob es sich bei der vorgesehenen Anwendung ionisierender Strahlung oder radioaktiver Stoffe um ein anerkanntes bzw. etabliertes Verfahren nach den Erfordernissen der medizinischen Wissenschaften handelt. Ist dies nicht der Fall, hat der Arzt die Erforderlichkeit besonders zu begründen und gesondert zu dokumentieren, vgl. § 85 Abs. 1 S. 2 Nr. 1 StrlSchG. ${ }^{13}$

Insbesondere bedeutsam sind zudem die Pflichten, die eine Befragung des Patienten vor der Anwendung der ionisierenden Strahlung vorsehen, so wie dies in den vorstehenden Ausführungen stellenweise bereits angeklungen ist. Der übergeordnete Zweck der rechtfertigenden Indikation liegt darin, unnötige Strahlenexpositionen zu vermeiden und die Strahlenbelastung insgesamt gering zu halten, §119 Abs. 3 S. 1 a.E.

12 Vgl. ebd.

13 Vgl. auch Begründung zur Verordnung, BR-Drs. 423/18, S. $420 \mathrm{f}$
StrlSchV. Der Arzt hat zu diesem Zweck die verfügbaren Informationen über bisherige medizinische Erkenntnisse heranzuziehen und sich dabei ggf. auch des überweisenden Arztes oder Zahnarztes zu bedienen. Der Patient ist zudem hinsichtlich früherer bedeutsamer Anwendungen ionisierender Strahlung oder radioaktiver Stoffe zu befragen. Dass diese Befragung durch den Arzt geschehen müsste, ist ausweislich des Wortlauts des $§ 119$ Abs. 3 S. 2 StrlSchV nicht anzunehmen:

„Zu diesem Zweck [Anmerkung der Verfasser: Der hier in Bezug genommene Zweck ist die Vermeidung jeder unnötigen Strahlenexposition, s. o.] ist die zu untersuchende oder zu behandelnde Person über frühere Anwendungen ionisierender Strahlung oder radioaktiver Stoffe, die für die vorgesehene Anwendung von Bedeutung sein können, zu befragen."

Ähnlich wie $\S 124$ Abs. 1 StrlSchV trifft auch $\S 119$ Abs. 3 S. 2 StrlSchV keine Aussage darüber, wer die in der Vorschrift beinhalteten Pflichten zu erfüllen hat. Wie sich jedoch insbesondere aus dem Zusammenspiel der amtlichen Begründungen zu $§ 83$ Abs. 3 S. 4 StrlSchG und zu seiner Vorgängerregelung ergibt, entspricht es nicht dem Willen des Gesetzgebers, eine generelle ärztliche Pflicht zur persönlichen Untersuchung des Patienten zu normieren. Es wird deutlich, dass eine Änderung der bisherigen Rechtslage nach der RöV auch unter dem Regime von StrISchG und (neuer) StrlSchV durch den Gesetzgeber nicht intendiert ist. Daraus folgt, dass eine der Untersuchung vorausgehende Befragung grundsätzlich nicht durch den Arzt persönlich erfolgen muss, sondern an hinreichend qualifiziertes nicht-ärztliches Personal delegiert werden kann, wie dies bereits nach der Rechtslage unter der RöV der Fall war. ${ }^{14}$

Eine Pflicht zur persönlichen Untersuchung und ggf. auch Aufklärung über Risiken und Folgen der Anwendung ionisierender Strahlung ergibt sich jedoch ausnahmsweise dann, wenn die Befragung des Patienten oder die herangezogenen Informationen über ihn ergeben, dass er als Risikopatient zu behandeln ist. Dies gilt einerseits für

14 So auch Wigge/Loose, RöFo 2/2016, 218, 223 f.; RöFo 3/2016, 312, $313 \mathrm{ff}$
Patienten, die in der Vergangenheit bereits wiederholt gegenüber Strahlung in dem betreffenden Untersuchungsgebiet exponiert waren, andererseits und insbesondere für schwangere Patientinnen oder solche, bei denen eine Schwangerschaft nicht auszuschließen ist, $\S 120$ Abs. 1 S. 1 und Abs. 2 S. 1 StrISchV. Das vorgenannte gilt ebenso für stillende Patientinnen, §120 Abs. 1 S. 3 und Abs. 2 S. 2 StrlSchV.

Zusammengefasst folgt hieraus, dass die sonstigen strahlenschutzrechtlichen Vorgaben hinsichtlich der Stellung der rechtfertigenden Indikation keine grundsätzliche Pflicht des Arztes vorsehen, den Patienten persönlich zu untersuchen oder aber über spezifische Risiken der Strahlenanwendung aufzuklären, sofern sich keine Anhaltspunkte für die Zugehörigkeit zu einer der genannten Risikogruppen ergeben. In letzterem Fall ist die Dringlichkeit der Anwendung der ionisierenden Strahlung zu prüfen, vgl. § 120 Abs. 1 S. 2 StrlSchV. Damit sind die Fälle, in denen an die Rechtfertigung der Strahlenanwendung im Einzelfall besondere Anforderungen zu stellen sind, in der StrlSchV abschließend beschrieben. Für das Bedürfnis, diese erhöhten Sorgfaltsanforderungen des Arztes auch auf regelhafte Fälle der Strahlenanwendung auszudehnen, bestehen vor dem Hintergrund der sonstigen strahlenschutzrechtlichen Regelungen keine Anhaltspunkte.

\section{Zivilrechtliche Pflichten des Radiologen aus dem Behandlungsvertrag}

Fraglich ist jedoch, ob anderes aus der oben bereits zitierten Begründung zu $§ 124$ Abs. 1 StrlSchV folgt, die - in nicht eindeutiger Weise - auf zivilrechtliche Vorschriften verweist. An dieser Stelle ist es erforderlich, die Grundsätze über die Aufklärung nach den einschlägigen zivilrechtlichen Vorschriften im Überblick darzustellen und diese daraufhin zu überprüfen, ob sie sich überhaupt zu den oben dargestellten Ergebnissen und zu den entsprechenden strahlenschutzrechtlichen Vorgaben in Widerspruch setzen.

\section{a. Patientenrechtegesetz}

Mit dem Gesetz zur Verbesserung der Rechte von Patientinnen und Patienten - 
Patientenrechtegesetz vom 20.02.2013 ${ }^{15}$ das am 26.02.2013 in Kraft getreten ist, wurden die wesentlichen richterrechtlich bereits verstetigten Pflichten des Behandlers im Behandlungsverhältnis kodifiziert ${ }^{16}$. Im Zuge dessen fanden auch die bereits zuvor in der Rechtsprechung gefestigten Grundsätze der Selbstbestimmungsaufklärung in $\S 630$ e BGB und der therapeutischen Aufklärung bzw. Sicherungsaufklärung in $\S 630 c$ Abs. 2 BGB Eingang.

\section{b. Pflicht zur Risikoaufklärung}

Nach gefestigter Rechtsprechung stellt auch der lege artis ausgeführte ärztliche Heileingriff als Eingriff in die körperliche Unversehrtheit des Patienten grundsätzlich eine objektiv tatbestandliche Körperverletzung dar. Diese bedarf zu ihrer Rechtfertigung der Einwilligung des Patienten. Dem trägt die Vorschrift des §630d Abs. 1 S. 1 BGB Rechnung, die für den Regelfall voraussetzt, dass der Behandelnde vor Durchführung einer medizinischen „Maßnahme“ die Einwilligung des Patienten einzuholen hat. Damit der Patient seine Einwilligung wirksam im Sinne eines informed consent, $d$. h. im Bewusstsein über Gegenstand und Tragweite seines Rechtsgutsverzichts, erteilen und damit sein Selbstbestimmungsrecht ausüben kann, ist er rechtzeitig vor Durchführung der Maßnahme durch den Arzt ordnungsgemäß gemäß §630d Abs. 2 BGB i. V. m. §630e Abs. 1 bis 4 BGB aufzuklären.

Der §630e Abs. 1 BGB gestaltet die Pflicht zur Durchführung dieser sogenannten Selbstbestimmungsaufklärung ${ }^{17}$ des $\mathrm{Pa}$ tienten in folgender Weise aus:

„Der Behandelnde ist verpflichtet, den Patienten über sämtliche für die Einwilligung wesentlichen Umstände aufzuklären. Dazu gehören insbesondere Art, Umfang, Durchführung, zu erwartende Folgen und Risiken der Maßnahme sowie ihre Notwendigkeit, Dringlichkeit, Eignung und Erfolgsaussichten im Hinblick auf die Diagnose oder die Therapie. Bei der Aufklärung ist auch auf Alternativen zur Maßnahme hinzuweisen, wenn mehrere medizinisch

15 BGBI.I, S. 277.

16 Vgl. BR-Drs. 312/12, S. 30, 34.

17 Vgl. u. a. Katzenmeier, in: Laufs/Katzenmeier/ Lipp, Arztrecht, 8. Auflage 2021, Kap. V, Rn. $14 \mathrm{f}$. gleichermaßen indizierte und übliche Methoden zu wesentlich unterschiedlichen Belastungen, Risiken oder Heilungschancen führen können. “

Die Pflicht zur Aufklärung trifft nach §630e Abs. 2 Nr. 1-3 BGB den Arzt persönlich bzw. einen ausreichend qualifizierten Kollegen, wobei die Erfüllung dieser Aufklärungsverpflichtung im persönlichen Gespräch mit dem Patienten mündlich, rechtzeitig und verständlich zu erfolgen hat. Eine Delegation an nicht-ärztliches Personal ist ausgeschlossen. ${ }^{18}$

Allerdings besteht das Erfordernis der Einholung einer Einwilligung des Patienten grundsätzlich nur, wenn ein „Eingriff“ insbesondere in die ausdrücklich genannte körperliche Integrität erfolgt oder aber zumindest persönlichkeitsrechtlich geschützte Interessen des Patienten durch die Maßnahme betroffen sind. ${ }^{19}$ Hierzu haben Wigge und Loose bereits eingehend beschrieben, dass die Frage nach dem Eingriffscharakter sich im Wesentlichen danach beantwortet, ob ein Schaden an Körper und Gesundheit des Patienten auch nachweislich kausal auf die einmalige Strahlenanwendung zurückzuführen ist. ${ }^{20}$ In Bezug auf ein Aufklärungserfordernis hinsichtlich sogenannter stochastischer Strahlenschäden, d.h. solcher Schäden, die auf einer unzureichenden Reparatur der von der Strahlenanwendung beschädigten Zellen beruhen und neoplastische Veränderungen und Erbkrankheiten hervorrufen können, haben Wigge und Loose herausgestellt:

„Bei einer Einzelperson ist es daher im Ergebnis nicht möglich, einen kausalen Zusammenhang zwischen einer vorausgegangenen Strahlenexposition und einer klinisch manifestierten Krebserkrankung nachzuweisen, weil ein durch Strahlung verursachtes Karzinom klinisch nicht von denjenigen zu unterscheiden ist, die durch andere natürliche oder zivilisatorische Einflüsse hervorgerufen wurden. “21

Diese Feststellung wird auch durch höchstrichterliche Rechtsprechung gestützt. Hervor-

18 Vgl. u. a. Spickhoff in: Spickhoff, Medizinrecht, 3. Auflage 2018, BGB §630e Rn. 3 ff.

19 Vgl. BT-Drs. 17/10488, S. 23.

20 Wigge/Loose, RöFo 2/2016, 218, 220; vgl. auch RöFo 3/2016, 312, 314

21 Wigge/Loose, RöFo 2/2016, a. а. O. zuheben ist vor allem das Urteil des Bundesgerichtshofs $(\mathrm{BGH})$ vom 12.12.198922. In dem der Entscheidung zu Grunde liegenden Sachverhalt hatte sich der BGH mit der Frage zu befassen, ob zum Zeitpunkt der Durchführung der streitgegenständlichen Telekobaltbestrahlung - ein Verfahren der Strahlentherapie - eine Pflicht des die Behandlung durchführenden Arztes bestanden hatte, den Patienten über das Risiko von Strahlenschäden aufzuklären. Der Senat stellt in den Entscheidungsgründen des Urteils zwar heraus, dass im Grundsatz auch über sehr seltene, die Lebensführung des Patienten aber im Falle des Eintritts stark belastende, gefährliche Nebenwirkungen der angewandten Strahlentherapie-Methode aufzuklären sei. Jedoch machte das Gericht die bedeutsame Einschränkung, dies gelte

„[...] nur dann, wenn nach dem medizinischen Erfahrungsstand im Zeitpunkt der Behandlung ein solches Risiko bekannt und mit seinem Eintritt zu rechnen gewesen ist. "23

Das Bestehen eines solchen Risikos, mit dessen Eintritt zu rechnen gewesen war, erkannte der Senat in dem verhandelten Fall nicht. ${ }^{24}$ In den Entscheidungsgründen wird ausgeführt:

„Nach Ansicht des erkennenden Senates kann auch nicht [...] vor einer Strahlentherapie wegen ihrer spezifischen Gefahren verlangt werden, daß der behandelnde Arzt seinen Patienten darauf hinweist, es könne bei höheren Strahlendosen als für die Behandlung geplant in seltenen Fällen zu Strahlenschäden kommen. Solche rein theoretisch bleibende Erörterungen über Risiken, die bei anderer Behandlungsstrategie bekannt sind, sind in aller Regel ebensowenig wie allgemeine Überlegungen dazu, daß der Eintritt bislang unbekannter Komplikationen in der Medizin wohl nicht ganz auszuschließen sind, für die Entscheidungsfindung des Patienten von Bedeutung. Sie würden ihn im Einzelfall sogar nur unnötig verwirren und beunruhigen. “25

22 BGH, Urteil vom 12.12.1989, Az.: VI ZR 83/89, MedR 1990, 135 f. = NJW 1990, 1528f., zitiert nach juris.

23 Vgl. BGH, a. a. O., juris Rn. 10.

24 Vgl. BGH, a. a. O., juris Rn. 13.

25 BGH, a. a. O., juris Rn. 14. 
Aus dieser höchstrichterlichen Rechtsprechung lässt sich ableiten, dass, gemessen an den im eben dargestellten Urteil aufgestellten Grundsätzen, ein Patient über die allenfalls theoretisch denkbaren Strahlenschäden, die er aufgrund einer einzelnen durchgeführten Röntgenaufnahme erleiden könnte, auch nach zivilrechtlichen Grundsätzen nicht aufzuklären ist.

Zwar ist der $\mathrm{BGH}$ in seinem Urteil vom 03.12.199726 zu der Feststellung gelangt, dass im Falle der Durchführung medizinisch nicht indizierter Röntgenaufnahmen der Tatbestand der gefährlichen Körperverletzung erfüllt sein könnte. Zu der Feststellung einer Körperlverletzung war der Senat deswegen gelangt, weil der angeklagte Arzt in zahlreichen Fällen in exzessiver Weise geröntgt hatte. ${ }^{27}$ Diese Feststellung ist aus guten Gründen zu kritisieren. Jedenfalls aber, und das ist für die eingangs dargestellte Problematik von besonderer Relevanz, stellt der 2. Strafsenat des BGH auch in dieser Entscheidung heraus, dass

„[d]ie einmalige, kurzzeitige oder nur gelegentlich wiederholte ordnungsgemäße Anwendung von Röntgenstrahlen [...] in der Regel noch nicht als Körperverletzung zu beurteilen sein [mag]. “28

Selbst wenn also, wie die Begründung zu $\S 124$ Abs. 1 StrISchV andeutet, auf die Vorschriften über die Aufklärung abzustellen ist, um den Regelungsinhalt der dort geregelten Informationspflicht näher zu bestimmen, muss man zu dem Ergebnis kommen, dass eine grundsätzliche Pflicht zur Aufklärung über Folgen und Risiken der Durchführung einer einzelnen Röntgenaufnahme nach den für die Aufklärung im Zivilrecht maßgeblichen Grundsätzen des §630e Abs. 1 S. 1 BGB nicht besteht, da ein Eingriff in ein Rechtsgut des Patienten mit der Durchführung einer einzelnen Röntgenaufnahme grundsätzlich nicht verbunden ist.

Im Wesentlichen ist dies damit zu begründen, dass ein Kausalzusammenhang zwischen der Strahlendosis, die bei einer einzelnen,

26 BGH, Urteil vom 03.12.1997, Az.: 2 StR 397/97, BGHSt 43, $346 \mathrm{ff}$. = MedR 1998, $326 \mathrm{ff}$., zitiert nach juris.

27 BGH, a. a. O., juris Rn. 21.

28 Ebd. ordnungsgemäß durchgeführten Röntgenaufnahme zur Anwendung kommt, und etwaigen aus der Aufnahme resultierenden stochastischen Strahlenschäden ohnehin nicht klinisch nachweisbar ist. Im Übrigen aber ist die angeführte höchstrichterliche Rechtsprechung zum Aufklärungserfordernis vor der Durchführung von Maßnahmen der Strahlentherapie zu beachten. Wenn schon hinsichtlich der weit eingriffsintensiveren Maßnahmen der Strahlentherapie, bei der ungleich höhere Strahlendosen als bei der Durchführung von Röntgenuntersuchungen zur Anwendung kommen, ein Aufklärungserfordernis hinsichtlich Folgen und Risiken der angewandten ionisierenden Strahlung nicht besteht, so gilt dies erst Recht für Röntgenuntersuchungen. ${ }^{29}$

Wie aufgezeigt werden konnte, gehen die grundsätzlich weiter gefassten Aufklärungserfordernisse, wie sie sich nach den einschlägigen zivilrechtlichen Vorschriften darstellen, jedenfalls im Falle von Röntgenuntersuchungen im Ergebnis nicht weiter, als es die Vorschriften des Strahlenschutzrechts tun. Unabhängig davon ist ergänzend anzumerken, dass die spezielleren öffentlich-rechtlichen Vorschriften des Strahlenschutzrechts geeignet sind, die zivilrechtlichen Vorschriften zu derogieren, d. h. zu überlagern, soweit diese ihnen widersprechen. Die Vorgaben des Strahlenschutzrechts wirken daher, gerade mit Blick auf die abschließende Regelung von besonderen Risikokonstellationen bei der Anwendung ionisierender Strahlen und radioaktiver Stoffe, auch in die zivilrechtlichen Vorgaben zu den Aufklärungserfordernissen hinein.

Im Ergebnis besteht daher keine grundsätzliche zivilrechtliche Pflicht des Radiologen, vor der Durchführung einer einzelnen, indizierten Röntgenaufnahme eines regelhaften Patienten persönlich, mündlich und gesondert über das Risiko der Anwendung ionisierender Strahlung im Rahmen des $§ 630 \mathrm{e}$ Abs. 1 S. 1 und Abs. 2 BGB aufzuklären.

\section{c. Informationspflicht}

In $\S 630$ c Abs. 2 S. 1 BGB finden sich die dem Behandelnden auferlegten Informationspflichten, die vor dem Inkrafttreten

29 Zu alledem Wigge/Loose, RöFo 2/2016, 218, $219 \mathrm{ff}$. des Patientenrechtegesetzes in der Rechtsprechung als Sicherungsaufklärung bzw. therapeutische Aufklärung anerkannt waren. ${ }^{30}$ Nach dem Wortlaut ist der Behandelnde hiernach dazu verpflichtet,

„[...], dem Patienten in verständlicher Weise zu Beginn der Behandlung und, soweit erforderlich, in deren Verlauf sämtliche für die Behandlung wesentlichen Umstände zu erläutern, insbesondere die Diagnose, die voraussichtliche gesundheitliche Entwicklung, die Therapie und die zu und nach der Therapie zu ergreifenden Maßnahmen. “

Die vorgenannte Informationspflicht dient der Sicherung des Therapieerfolges durch Herstellung oder Erhöhung der Patientencompliance. Auch die Begründung zu § 124 Abs. 1 StrISchV verweist auf den vorgenannten Satz 1. Dabei ist zu beachten, dass eine entsprechende Information des Patienten nicht grundsätzlich und zwingend, sondern nur sofern erforderlich zu erfolgen hat. Zudem könnte man, selbst wenn die im Strahlenschutzrecht geregelten Informationspflichten wie die zivilrechtlichen Informationspflichten aus $\S 630$ c Abs. 2 S. 1 BGB zu behandeln wären, aufgrund des Wortlauts des $\S 124$ Abs. 1 StrlSchV nicht ableiten, dass den Arzt die Informationspflicht träfe, da dieser regelmäßig nicht Strahlenschutzverantwortlicher ist.

\section{Adressat der Informations- pflichten gemäß §124 Abs. 1 StrISchV}

Wie soeben angedeutet, spricht auch der Wortlaut des $\S 124$ Abs. 1 StrlSchV selbst gegen eine Verpflichtung des Arztes zur persönlichen Aufklärung oder Information über die Risiken der Anwendung ionisierender Strahlung: Nach eindeutigem Wortlaut der Vorschrift ist der Strahlenschutzverantwortliche Adressat der Informationspflichten. Dieser muss kein Arzt sein, wie bereits die innere Systematik des $§ 120$ StrlSchV zeigt: Während dessen Absätze 1 und 2 sich eindeutig an den die ionisierende Strahlung oder radioaktive Stoffe anwendenden Arzt oder Zahnarzt wenden, ist der Absatz 3 des $\S 120$ StrISchV in vergleichbarer Weise wie

30 Spickhoff, a. a. O., BGB §630c Rn. 12. 
der § 124 StrISchV formuliert. Auch hier wird die Formulierung, „[d]er Strahlenschutzverantwortliche hat dafür zu sorgen, dass [...]“ gebraucht. Würde es sich bei dem die Maßnahme durchführenden Arzt um den Strahlenschutzverantwortlichen handeln, wäre eine begriffliche Differenzierung hinsichtlich der Adressaten in den Absätzen ohne erkennbaren Sinn.

Ausweislich §69 Abs. 2 S. 1 StrlSchG muss es sich bei dem Strahlenschutzverantwortlichen nicht einmal um eine natürliche Person handeln, wobei im Falle juristischer Personen regelmäßig deren gesetzliche Vertreter die Aufgaben des Strahlenschutzverantwortlichen wahrzunehmen haben. An die Fachkunde des Strahlenschutzverantwortlichen werden - in Abgrenzung zur Person des Strahlenschutzbeauftragten keine weiteren Anforderungen gestellt. Die Vorschrift des $§ 124$ Abs. 1 StrlSchV richtet sich demnach im Ergebnis einerseits bereits nicht an den Arzt, der ionisierende Strahlung oder radioaktive Stoffe am Patienten anwendet, andererseits ist von einer umfassenden Delegierbarkeit der Informationspflichten des Strahlenschutzverantwortlichen auszugehen, die auch nicht-ärztliches Personal einschließt.

\section{Praxishinweis und Fazit}

Als wesentliches Ergebnis ist daher festzuhalten, dass für den Radiologen auch nach den Vorgaben des neuen Strahlenschutzrechts, also nach dem StrlSchG und der StrISchV, keine Pflicht besteht, den Patienten vor der Durchführung einer Röntgenaufnahme über das Risiko der Strahlenanwendung gesondert im persönlichen Gespräch aufzuklären. Mit der Vorschrift des $§ 124$
Abs. 1 StrlSchV ist nicht intendiert, die Grundsätze zu verschärfen, die aus den anderen strahlenschutzrechtlichen Vorschriften, insbesondere denen zur rechtfertigenden Indikation, ableitbar sind. Nach der Verordnungsbegründung ist $§ 124$ StrlSchV mit den Informationspflichten des Behandlers aus $\S 630$ c Abs. 2 S. 1 BGB vergesellschaftet, sodass bereits nach dem Wortlaut des $\S 630$ c BGB - und entgegen dem Wortlaut des $\S 630$ e Abs. 2 S. 1 Nr. 1 BGB - jedenfalls kein Formerfordernis für die Erfüllung dieser Informationspflicht besteht.

Für die radiologische Praxis ist gleichwohl anzuraten, die Einholung einer Einwilligung in die Röntgenuntersuchung gemäß § 630d BGB auf einem durch den Patienten unterschriebenen Aufklärungsbogen zu sichern. Dies gilt einerseits aus Gründen der Beweissicherung vor dem Hintergrund eines (theoretisch) möglichen Arzthaftungsprozesses, auch wenn regelmäßig kein Einwilligungserfordernis hinsichtlich einer einzelnen Röntgenuntersuchung besteht. Andererseits ermöglicht ein derartiger Aufklärungsbogen, wenn dieser Angaben über die Risiken einer Strahlenexposition beinhaltet, dem Strahlenschutzverantwortlichen gleichzeitig, ohne Mehraufwand und damit kostenneutral, seine strahlenschutzrechtlichen Informationspflichten zu erfüllen. Schließlich bietet ein Aufklärungsbogen Gelegenheit, ggf. bestehende Anhaltspunkte für die Zugehörigkeit des Patienten zu einer Risikogruppe zu erfragen.

Feststeht, dass der Arzt den Patienten, bei dem er eine Röntgenuntersuchung durchzuführen beabsichtigt, nur dann tatsächlich persönlich untersuchen muss, wenn Anhaltspunkte für ein erhöhtes Risiko hinsichtlich der
Anwendung ionisierender Strahlung vorliegen. Ein solches erhöhtes Risiko ist vor allem bei häufiger Strahlenanwendung in der Vergangenheit und bei einer (möglichen) Schwangerschaft einer Patientin gegeben. Der Radiologe muss sich jedoch so in räumlicher Nähe aufhalten, dass ihm eine derartige Untersuchung jedes Patienten vor der Durchführung der Röntgenaufnahme zumindest in jedem Falle möglich ist. Die der Röntgenuntersuchung vorausgehende Befragung des Patienten zu dem Zweck, ein erhöhtes Risiko festzustellen, kann jedoch grundsätzlich von dem hinreichend qualifizierten nicht-ärztlichen Personal des Radiologen vorgenommen werden.

Als Fazit kann festgehalten werden, dass mit der Neuordnung des Strahlenschutzrechts Ende des Jahres 2018 keine inhaltliche Änderung der Rechtslage hinsichtlich der Aufklärungspflichten und der übrigen vorgenannten Pflichten des Radiologen vor und bei Durchführung einer einzelnen indizierten Röntgenaufnahme einhergegangen ist.

Prof. Dr. Peter Wigge

Rechtsanwalt

Fachanwalt für Medizinrecht

Philip Steuwer

Rechtsanwalt

Rechtsanwälte Wigge

Scharnhorststraße 40

48151 Münster

Telefon: (0251) 53 595-0

Telefax: (0251) 53 595-99

E-Mail: kanzlei@ra-wigge.de

www.ra-wigge.de 